\title{
Trazando un mapa de los medios digitales: Uruguay
}

\author{
Giorgina Santangelo
}

Open Society Foundations, noviembre de 2013.

Editor: Fernando Bermejo (editor regional para Open Society Foundations). Número de páginas: 97.
En los últimos quince años, el avance de las tecnologías de la información y comunicación (TIC), como sistemas tecnológicos mediante los cuales se recibe, manipula y produce información, ha contribuido a un aumento cuantitativo de la conectividad entre las personas y del flujo de información circulante en el mundo. La utilización de las TIC requiere un proceso de digitalización de la información producida que permita recodificar flujos de información y comunicación en diferentes formatos -textos, videos, sonido, voz u otros medios-. En este sentido, el surgimiento de la sociedad de la información no se da simplemente por la inversión en infraestructura y en producción tecnológica, sino que además requiere digitalizar para abrir la posibilidad de los intercambios de información.

En el contexto de estas nuevas dinámicas y del desarrollo del campo de la comunicación, la información se transforma en un recurso de poder, y la opacidad en la difusión de información de las organizaciones públicas o privadas es interpelada ante la demanda de un aumento de transparencia. El acceso a la información se vuelve un desafío en las democracias del siglo XXI, puesto que fortalece la capacidad ciudadana de participar en decisiones que afectan sus vidas y de fiscalizar el desempeño de las instituciones.

En este escenario, promover un entorno mediático (estructura, contenido e impacto de los medios en las personas) libre, independiente y pluralista se torna indispensable para fortalecer este régimen de gobierno.

El informe país Trazando un mapa de los medios digitales: Uruguay, a cargo de Rosario Radacovich, Santiago Escuder, Julia Morales y Matías Ponce, da cuenta de esta realidad. Se estructura como un informe técnico y es parte de un proyecto de investigación macro que lleva adelante la Open Society Foundations en más de sesenta países, cuyo objetivo es evaluar el progreso de la digitalización y su impacto en la libertad de prensa, el pluralismo, el acceso de los ciudadanos a las noticias, la información de calidad, la trasparencia y la fiscalización, la independencia editorial y la libertad de expresión, entre otros elementos indispensables para el sostenimiento del régimen democrático.

A la luz de estos valores, el informe Uruguay evalúa las oportunidades y los riesgos que presentan para los medios de comunicación tres procesos específicos: la transición de la transmisión analógica a la digital, el crecimiento de nuevas plataformas de medios de comunicación como fuente de noticias y la convergencia de la transmisión tradicional con las telecomunicaciones.

Trazando un mapa de los medios digitales: Uruguay se estructura en ocho capítulos. El primero da cuenta del cambio en el consumo de medios tradicionales (televisión, radio y prensa) hacia un uso cada vez más intenso de los medios digitales, en particular en la franja etaria de 16 a 29 años. Se observa una migración de lectores de la prensa escrita a la digital, que en algunos puntos supera a la primera.

Estos cambios se atribuyen a la expansión de Internet como resultado de programas gubernamentales para lograr la equidad digital, como el Plan Ceibal - programa nacional que otorga una computadora personal a estudiantes y maestros de escuelas públicas (véase www.ceibal.edu.uy)-, y a la alta penetración de la telefonía móvil.

El lector de prensa digital es predominantemente montevideano y lee en promedio 20 minutos menos por semana que el lector de prensa escrita, pero, según 
los investigadores, no tiene a su disposición mayor diversidad en el contenido de noticias, ya que, a excepción de dos portales noticiosos, el contenido digital es el mismo que el impreso. Por otra parte, los programas de noticias de radios AM conservan la lealtad de los oyentes en los estratos más altos de la población, y los programas de noticias de los canales privados alcanzan mayor nivel de audiencia, manteniendo a la televisión como el principal proveedor.

En el capítulo 2 se brinda un panorama del servicio de medios públicos y estatales. Se señalan algunas iniciativas que dan cuenta de los avances en este tipo de medios, pero también se indican algunos aspectos pendientes. En las radios administradas por el Estado se reconoce la reorganización de la estructura de programación y la capacitación de personal, a pesar de que todavía tienen bajos índices de sintonía. En el período 2005-2007 se resalta la innovación de la programación del Canal 5, Televisión Nacional Uruguay (TNU), que incluyó programas de interés general y cultural, contribuyendo significativamente a la diversidad de contenidos.

Se señala la necesidad de mejorar el sistema administrativo y gerencial. En términos legislativos, se destaca el proyecto de ley aprobado en enero de 2013 que crea el Servicio de Comunicación Audiovisual Nacional (SCAN), compuesto por TNU y el Instituto del Cine y Audiovisual del Uruguay (ICAU). El SCAN coordina el diseño de un programa nacional de comunicaciones audiovisuales, lo que a juicio de los investigadores es un avance hacia una mejor organización y gestión de los medios públicos de comunicación.

Otra iniciativa jurídica destacada es la Ley de Servicios de Comunicación Audiovisual (mayo de 2013), que cuenta con media sanción parlamentaria. Algunos de los temas significativos abordados en el proyecto de ley son las limitaciones a la propiedad de servicios de comunicación, en orden a restringir monopolios, las limitaciones sobre compañías de televisión por cable en términos de participación en el mercado, ciertas restricciones sobre el tipo de programación y publicidad en los espacios de protección especial, y derechos de acceso de contenido a grupos minoritarios, entre otros.

Se menciona también la presencia de Tevé Ciudad, desde 1996, como canal público administrado por la Intendencia de Montevideo. Esto crea un nuevo modelo de televisión pública, enfocada en producciones culturales y temas locales, de cultura popular. Sin embargo, el canal aún no ha logrado instalarse en el público y mejorar sus niveles de audiencia.

Finalmente, se espera que la digitalización iniciada con la aprobación del Decreto sobre Televisión Digital sea una oportunidad para el fortalecimiento y la expansión de la televisión pública.

El capítulo 3 analiza la capacidad de generación de contenido por los usuarios de Internet en el país, a nivel individual. Esta herramienta es empleada para la búsqueda de información, las conversaciones por chat, el correo electrónico y las redes sociales. Solo el $6 \%$ de los usuarios de Internet cuenta con un blog y el mismo porcentaje comenta periódicamente acerca de los blogs. Si bien las redes sociales son una plataforma importante para el consumo de noticias e información, la investigación muestra que la herramienta blogging, que permite el intercambio de comentarios y opiniones a través de portales electrónicos, es de uso marginal entre los usuarios uruguayos.
Giorgina Santangelo:: Departamento de Comunicación de la Facultad de Ciencias Humanas de la Universidad Católica del Uruguay. gsantang@ucu.edu.uy 
El ejercicio de ciudadanía a través de la participación en movimientos sociales organizados cuenta con una larga tradición en Uruguay. Sin vinculación formal con el Estado, los partidos políticos o instituciones religiosas, las personas utilizan las plataformas virtuales para la organización y la movilización social. Con base en estas observaciones, el informe señala que, pese a la digitalización y al incremento en el número de fuentes de noticias, la oferta noticiosa en Internet ha sido bastante escasa.

El capítulo 4 estudia el impacto de la digitalización en el modelo empresarial de producción de los medios de comunicación tradicionales. Las salas de redacción de los medios digitales están integradas por entre cinco y diez periodistas, la mitad o menos del personal que emplearía un diario tradicional. Por ello, los periodistas de medios digitales deben ser más productivos en períodos más breves y con menos recursos. A su vez, los profesionales que trabajan en los medios digitales suelen ser graduados en busca de experiencia, lo que hace que los medios digitales sufran mayor movilidad de personal que los medios impresos tradicionales.

Estos son algunos de los factores mencionados cuando se argumenta que la estructura de inversión no ha sufrido grandes cambios y se sigue haciendo periodismo 1.0. El informe advierte que la digitalización ha transformado las rutinas de los periodistas, ha posibilitado el acceso a nuevas fuentes, lo que se traduce en mayor calidad de la información en los medios, y ha contribuido a la introducción de nuevos temas que no tenían cabida en los medios tradicionales -como violencia de género, discriminación o situación de niños y adolescentes-. Sin embargo, los periodistas enfrentan el reto de trabajar con un abordaje diferente en un tiempo más apremiante que el de la prensa tradicional.

Las políticas de asignación del espectro se abordan en el capítulo 5. Allí se señala que los medios de comuni- cación tradicionales en el país han estado concentrados en manos de importantes grupos mediáticos. Durante la última década han aparecido nuevas entidades reguladoras y un marco legal ${ }^{1}$ que, según los especialistas, puede transformar e instituir un nuevo orden de asignación del espectro. La regulación en la asignación de frecuencias fortalece la supervisión y establece criterios para otorgar licencias de radio, televisión y medios comunitarios. En este sentido, el informe valora la creación de institucionalidad y marcos que sientan las bases de un posible cambio en el sistema de medios uruguayo.

El capítulo 6 analiza algunos temas legales vinculados a la propiedad de los medios en el país. Los medios de comunicación en Uruguay han estado dominados por tres grupos empresariales, que además están unidos a operadores locales de pequeña escala, lo cual dificulta la emergencia de nuevos actores en el mercado. En la capital, la televisión de señal abierta con alcance nacional está controlada por tres conglomerados que además controlan cuatro de las cinco licencias de televisión para abonados y la distribución de todas las estaciones de televisión abierta y servicios en los departamentos. Esta situación ubica a Uruguay como uno de los países de América Latina con más alta concentración en la propiedad de los medios de comunicación.

Aunque todos los operadores de medios audiovisuales deben registrarse en la Unidad Reguladora de Servicios en Comunicación (URSEC), donde se incluyen datos personales de los propietarios y representantes legales de las compañías, el frecuente uso de testaferros afecta la transparencia de esta información.

Los autores tienen expectativas de que la aplicación del Decreto de Televisión Terrestre Digital, que establece nuevas reglas sobre la propiedad, permita la competencia en el sector de televisión privada, así como el ingreso de nuevos actores. 
En el capítulo 7 se reflexiona sobre este decreto, que define nuevos mecanismos regulatorios para la asignación de frecuencias. Asimismo, la creación del Consejo Honorario de Radios Comunitarias y la Comisión Honoraria Asesora Independiente representa un esfuerzo por incluir a organizaciones de la sociedad civil en las discusiones sobre políticas de comunicación.

El capítulo 8 presenta las conclusiones del informe. El reforzamiento del marco regulatorio relacionado con el espectro de transmisiones y el diseño de políticas de comunicación se destacan como lo más trascendental en orden a fortalecer las condiciones democráticas para el sistema de medios de comunicación en Uruguay. Sin embargo, se advierte que los nuevos actores y las propuestas opuestas al sistema predominante aún no tienen cabida.

En suma, Trazando un mapa de los medios digitales: Uruguay constituye una referencia para quienes toman decisiones de políticas públicas, académicos, periodistas, empresarios de medios, editores, entidades reguladoras y autorreguladoras y la sociedad civil en general, ya que, con una triangulación de datos de fuentes primarias y secundarias, diagnostica el impacto de la digitalización en el país. Además, el hecho de contener la primera medición en su área lo transforma en una línea de base que permitirá monitorear la evolución del sistema de medios en Uruguay en la era digital.

Por otra parte, el informe constituye un antecedente relevante para el desarrollo de otras líneas de investigación, como el proyecto "Desarrollo mediático: Marco para evaluar el desarrollo de los medios de comunicación social”, que en Uruguay está a cargo de un equipo de investigadores y docentes de la Universidad de la República (LICCOM y ObservaTIC - Facultad de Ciencias Sociales), de la Universidad Católica del Uruguay (Cátedra UNESCO de Comunicación), de la
Universidad ORT (Facultad de Comunicación), de la Universidad de Montevideo (Facultad de Comunicación) y de la Universidad del Trabajo del Uruguay (Escuela Superior de Comunicación Social).

Este proyecto -idea original del Programa Internacional para el Desarrollo de la Comunicación (PIDC, UNESCO, 2008)- es una propuesta integral de indicadores que analizan los principales aspectos de la ecología mediática en determinado contexto nacional, por lo que requiere un anclaje en cada país. En la actualidad existen algunos antecedentes del estudio en Egipto, Túnez, Mozambique y Ecuador. En Uruguay el proyecto comenzó a gestarse a fines del 2012.

Una segunda línea de acción que ha surgido a consecuencia del estudio Trazando un mapa de los medios digitales: Uruguay está orientada a la implementación del proyecto "Gender Sensitive Indicators for Media: Framework of indicators to gauge gender sensitivity in media operations and content", de la UNESCO, cuyo objetivo es medir la sensibilidad de los medios de comunicación uruguayos frente a la problemática de género. La batería de indicadores está acotada tanto a relevar actitudes en operadores como aspectos cuantitativos (número de mujeres que ocupan cargos de toma de decisiones) y cualitativos (observación de roles asignados) en el contenido de la programación en medios de comunicación en Uruguay. Cabe destacar que esta dimensión es señalada como una línea poco desarrollada por los informes de diagnóstico tanto de la Asociación de la Prensa Uruguaya (APU) como de las organizaciones sociales especializadas en el tema.

En consecuencia, para el año 2015 se espera haber construido un mapa del entorno mediático y exponer la capacidad de los medios para promover una cultura en equidad de género. Además, se espera que sean más independientes, pluralistas, diversos y verdaderas plataformas de información democrática.:- 